\title{
The TLR2 Antagonist Staphylococcal Superantigen-Like Protein 3 Acts as a Virulence Factor to Promote Bacterial Pathogenicity in vivo
}

\author{
Kirsten J. Koymans ${ }^{a}$ Oliver Goldmann ${ }^{b}$ Christofer A.Q. Karlsson ${ }^{c}$ \\ Wiedjai Sital $^{\mathrm{a}}$ Robert Thänert $^{\mathrm{b}}$ Adinda Bisschop $^{\mathrm{a}}$ Manouk Vrieling ${ }^{\mathrm{a}}$ \\ Johan Malmström ${ }^{c}$ Kok P.M. van Kessela Carla J.C. de Haas ${ }^{a}$ \\ Jos A.G. van Strijp ${ }^{a}$ Eva Medinab \\ a Department of Medical Microbiology, University Medical Center Utrecht, Utrecht, The Netherlands; \\ b Infection Immunology Research Group, Helmholtz Centre for Infection Research, Braunschweig, Germany; \\ 'Division of Infection Medicine, Department of Clinical Sciences, Lund University, Lund, Sweden
}

\section{Keywords}

Staphylococcus aureus · Immune evasion · Innate immunity · Toll-like receptor $2 \cdot$ TLR2 antagonist · Staphylococcal superantigen-like protein 3

\begin{abstract}
Toll-like receptor (TLR) signaling is important in the initiation of immune responses and subsequent instigation of adaptive immunity. TLR2 recognizes bacterial lipoproteins and plays a central role in the host defense against bacterial infections, including those caused by Staphylococcus aureus. Many studies have demonstrated the importance of TLR2 in murine S. aureus infection. S. aureus evades TLR2 activation by secreting two proteins, staphylococcal superantigen-like protein 3 (SSL3) and 4 (SSL4). In this study, we demonstrate that antibodies against SSL3 and SSL4 are found in healthy individuals, indicating that humans are exposed to these proteins during S. aureus colonization or infection. To investigate the TLR2-antagonistic properties of SSL3 and SSL4, we compared the infection with wild-type and SSL3/4 knockout $S$. aureus strains in an intravenous murine infection model.
\end{abstract}

\section{KARGER}

(C) 2017 S. Karger AG, Basel
Direct evaluation of the contribution of SSL3/4 to infection pathogenesis was hindered by the fact that the SSLs were not expressed in the murine system. To circumvent this limitation, an SSL3-overproducing strain (pLukM-SSL3) was generated, resulting in constitutive expression of SSL3. pLukM-SSL3 exhibited increased virulence compared to the parental strain in a murine model that was found to be TLR2 dependent. Altogether, these data indicate that SSL3 contributes to S. aureus virulence in vivo. @ 2017 S. Karger AG, Basel

\section{Introduction}

Toll-like receptor 2 (TLR2) is an important pattern recognition receptor in the defense against bacterial infections [1]. TLR2 recognizes bacterial lipopeptides and lipoproteins together with its dimerization partners TLR1 and TLR6. Both ligand binding and subsequent dimerization with TLR1 or TLR6 are required for receptor functioning and downstream signaling through nuclear factor $(\mathrm{NF})-\kappa \mathrm{B}$, which leads to the production of proinflamma- 
tory cytokines [2]. TLR2 signaling is thought to be especially important in the initiation of innate immune responses, but it also plays a major role in the subsequent development of adaptive immunity to pathogens [1].

Staphylococcus aureus is a commensal bacterium, colonizing approximately $30 \%$ of the population, but it is also a highly successful pathogen, causing diseases ranging from mild skin infections to life-threatening invasive conditions such as sepsis [3]. It has emerged as a pathogen of global importance in humans and animals mainly through increases in antibiotic resistance [4]. Furthermore, the wide amount of secreted virulence factors, also called immune evasion molecules, play an important role in the pathogenicity of this bacterium [5].

S. aureus expresses approximately 50 different lipoproteins on its surface, representing approximately $2 \%$ of the staphylococcal proteome $[6,7]$. These lipoproteins are important for bacterial fitness and are for example involved in nutrient and iron uptake. S. aureus lipoproteins have also been shown to confer protection to the bacterium after phagocytosis, to ensure better survival and possible phagocytic escape [7]. On the other hand, these lipoproteins can also serve as pathogen-associated molecular patterns that can be recognized by host receptors such as TLR2, triggering inflammatory signaling pathways and thereby alerting the host immune system on the presence of $S$. aureus. In fact, appropriate host responses against $S$. aureus have been described to be dependent on the recognition of bacterial lipoproteins [8].

Although TLR2 is not a phagocytic receptor itself, it does play a role by providing a link between phagocytosis and inflammatory responses. TLR2 has also been described to contribute to neutrophil stimulation, and in several infection models TLR2 facilitated the recruitment of neutrophils $[9,10]$. The significance of TLR2-mediated immune responses during staphylococcal infections has been studied extensively in vivo in different murine models utilizing TLR2-knockout (KO) mice, but the outcomes vary vastly and seem to be related to the respective infection model. For intravenous infection, two separate studies have shown that TLR2-KO mice are more susceptible to lethal $S$. $a u$ reus infections $[11,12]$. In cutaneous $S$. aureus infection, TLR2-KO mice exhibited larger lesions and a slight increase in bacterial load as compared to wild-type (WT) mice; however, no defects in neutrophil recruitment were measured [13]. In murine models of $S$. aureus-induced brain abscess, the data vary: in one study TLR2 was required for survival [14], whereas another study measured no differences in survival between mouse strains; however, the expression of several immune-related factors, includ- ing proinflammatory cytokines, was attenuated in the TLR2-KO compared to the WT mice [15]. In a recent study by Blanchet et al. [16], TLR2-KO mice were more resistant to peritoneal S. aureus infection than WT mice. However, in the same study, these mice were more susceptible to pulmonary infection after intranasal inoculation. Another study, using a peritonitis model, found no differences in bacterial clearance between WT and KO mice in spleen and peritoneum [9]. Thus, although most studies indicate protective roles for TLR2 signaling during staphylococcal infections, there is no full consensus reached on the exact importance. It is likely that effects are highly depending on the bacterial dose and the route of infection.

S. aureus has developed strategies to circumvent recognition by TLR2. It secretes two small proteins that potently interfere with TLR2 signaling: staphylococcal superantigen-like protein 3 (SSL3) and 4 (SSL4) [17]. SSL3 and SSL4 are part of a larger, structurally related family of staphylococcal proteins that have diverse functions but are all involved in immune modulation [5]. SSL3 is the more potent inhibitor of the two, and the crystal structure of the complex of SSL3 and TLR2 revealed that SSL3 inhibits TLR2 through interfering with both lipopeptide binding and dimerization [18]. The secretion of two TLR2 inhibitors by $S$. aureus indicates that TLR2 inhibition is important for staphylococcal pathogenesis. However, little information is available regarding the regulation and expression of the SSLs. Although most ssl genes are found adjacent to each other on a single pathogenicity island, it is known that they all contain their own promoter region and are transcribed as monocistronic mRNAs, implying that they are regulated independently of each other [19]. Generally, S. aureus produces extremely low levels of SSLs under in vitro growth conditions. Upregulation of $s s l$ transcripts has been described under several conditions: in whole blood, upon contact with neutrophils, and under pore-mediated membrane stress [20-22]. SSL overproduction has been associated with increased virulence [19-21, 23].

The in vivo importance of the different SSLs still remains to be determined, and no research has been conducted to examine the role of SSL3 in particular. The ssl3 gene is present in almost all sequenced $S$. aureus strains $[24,25]$, which indicates that it plays a role at some point during infection or colonization. In the current paper, we aim to investigate the in vivo relevance of SSL3 in the pathogenicity of S. aureus. SSL3 is able to bind both murine and human TLR2 [17], and, therefore, its role in infection can be investigated in mouse models. However, the low expression of SSL3 by S. aureus in the murine
562

J Innate Immun 2017;9:561-573

DOI: $10.1159 / 000479100$
Koymans et al. 
system has posed an obstacle to these investigations. To circumvent this problem, we generated a genetically modified $S$. aureus strain that constitutively produces high levels of SSL3 by incorporating the highly active promoter of the leukocidin LukM upstream the gene encoding SSL3. The resulting overproducing strain produced high levels of SSL3 in vitro and exhibited a hypervirulent phenotype in mice after intravenous infection. This study contributes to our understanding of the mechanisms of staphylococcal immune evasion in vivo and illustrates the relevance of TLR2 signaling during $S$. aureus infections.

\section{Materials and Methods}

Cloning, Expression, and Purification of Recombinant SSL3 and SSL4

SSL3 (1-326, from S. aureus strain NCTC 8325, SAOUHSC_00386) and SSL4 (1-278, from S. aureus strain NCTC 8325, SAOUHSC_00389) were cloned, expressed, and purified as described previously [17]. In short, SSL3 was expressed in Escherichia coli Rosetta-gami(DE3)pLysS, with an N-terminal $\mathrm{His}_{6}$-tag and isolated from a HiTrap chelating HP column under nondenaturing conditions. Proteins were stored in PBS, and purity was determined by SDS-PAGE (purity $>95 \%$ ).

\section{Collection of Human Sera}

Venous blood was drawn from 36 different healthy adult volunteers and collected in 9-mL vacutainer blood tubes (BD) containing a clot activator. Clotting was allowed for $15 \mathrm{~min}$ at room temperature, and serum was collected by centrifugation at $2,080 \mathrm{~g}$ for $20 \mathrm{~min}$ at $4^{\circ} \mathrm{C}$ and subsequently stored at $-80^{\circ} \mathrm{C}$. Informed consent was obtained from all subjects in accordance with the Declaration of Helsinki. Approval from the Medical Ethics Committee of the University Medical Center Utrecht was obtained (METCprotocol 07-125/C approved on March 1, 2010).

ELISA for Antibody Titer Determinations in Human Serum

SSL3 and SSL4 were coated overnight in PBS $(10 \mu \mathrm{g} / \mathrm{mL})$ on a Nunc MaxiSorp 96-well ELISA plate (Sigma-Aldrich). The plate was blocked with $4 \%$ skimmed milk for $1 \mathrm{~h}$ at $37^{\circ} \mathrm{C}$ before adding human serum at different concentrations (from 0.001 to $10 \%$ ) from 36 different donors diluted in $1 \%$ skimmed milk in PBS containing $0.05 \%$ Tween 20 . Serum was incubated for $1 \mathrm{~h}$ at $37^{\circ} \mathrm{C}$ and anti-SSL3 and anti-SSL4 antibodies were detected using goat antihuman IgG-PO (1:6,000; Southern Biotech). ELISA was developed with freshly prepared tetramethylbenzidine, and the reaction was stopped with $4 \mathrm{~N}$ sulfuric acid. Anti-SSL3 and anti-SSL4 IgG titers were determined by frequency distributions of the log serum dilution reaching $50 \%$ of the optical density (OD) value after background subtraction.

\section{Bacterial Strains}

In all experiments, $S$. aureus strain $\mathrm{SH} 1000$ was used. The SSL3/4 double-KO (SSL3/4-KO) was created by designing primers recognizing $1,000 \mathrm{bp}$ upstream and $1,000 \mathrm{bp}$ downstream of the ssl 3 and ssl 4 genes, and these fragments were amplified from ge- nomic DNA of $S$. aureus strain SH1000. The following primers were used for amplification: SSL3 upstream forward $5^{\prime}$-CCGGGATTCTCTAATTATAATAGATTGGAG-3 and reverse 5'-CAGCTTCTATTTAGAGAATGTCATGAAATTGTATGCTCCAATCTATATTATATTC-3', and SSL4 downstream forward 5'-ATGACATTCTCTAAATAGAAGCTGTCATGACATTCTCTAAATAGAAGCTG- $3^{\prime}$ and reverse $5^{\prime}$-ATATGCGGCCGCTCAATGACGTCACTCATGCGATTTG-3'. The overlapping regions are shown in italics and the restriction sites are underlined. The fragments were merged with overlap extension PCR using Phusion HF polymerase (Bioke), and the final PCR product was ligated (T4 ligase) into a modified $\mathrm{pKOR}$ vector (pKOR-mcs), previously described by Stapels et al. [26] using EcoRI and NotI restriction sites. This product was cloned into E. coli DC10B, after which plasmid was isolated and analyzed for correct sequence. The vector was electroporated into S. aureus SH1000, and allelic replacement was induced [27]. Mutants were confirmed for deletion of ssl3 and ssl 4 by PCR and sequencing of the flanking regions.

To create the SSL3-overproducing strain, the promoter region of the leukocidin LukMF' was amplified by PCR from genomic DNA from bovine $S$. aureus strain S1444, as described previously [28]. The ssl3 gene was amplified from genomic DNA from the $S$. aureus strain SH1000. Both PCR products were annealed through overlapping regions that were introduced in the primers into a pCM29 vector [29] using Gibson Assembly (New England Biolabs). The following primers were used: LukM forward $5^{\prime}$-GCTTGCATGCCTGCAGGTCGACTCTAGAAAACGCACAGTTAATAAAAAG-3' and reverse $5^{\prime}$-gcaattgttctcattttcatAGTTTCACTTTCTTTCTCTTTA-3', and SSL3 forward 5'-TAAAGAGAAAGAAAGTGAAACTATGAAAATGAGAACAATTGC-3' ${ }^{\prime}$ and reverse $5^{\prime}$-GAAACAGCTATGACATGATTACGAATTCTTATTTTATATTCACTTCAATG-3'. The overlapping regions with the pCM29 vector sequence have been underlined. The overlapping regions for LukM and SSL3 are shown in italics and lowercase, respectively. This plasmid (pLukM-SSL3) was transformed into heat shock competent $E$. coli (DC10B), after which it was purified, and the sequence was confirmed to be correct. pLukM-SSL3 was introduced into S. aureus SH1000 through electroporation, and the presence of the plasmid was confirmed through PCR. The pLukMSSL3 strain was always grown in the presence of $10 \mu \mathrm{g} / \mathrm{mL}$ chloramphenicol to ensure plasmid retention.

\section{SDS-Page Gel for SSL3 Detection}

Supernatant from WT, SSL3/4-KO, and pLukM-SSL3 strains was harvested after overnight culture in Todd-Hewitt broth (THB); 2x sample buffer (+DTT) was added to the samples before boiling. Samples were loaded on $10 \%$ SDS-PAGE gels and run for $1 \mathrm{~h}$ at $200 \mathrm{~V} ; 10 \mu \mathrm{L}$ of purified SSL3 $(200 \mu \mathrm{g} / \mathrm{mL})$ was loaded as a control.

\section{Anti-TLR2 Antibody Competition Assay}

HEK cells stably expressing TLR2 (Invivogen) were maintained in DMEM, containing $10 \%$ fetal calf serum, $10 \mu \mathrm{g} / \mathrm{mL}$ blasticidin, $100 \mathrm{U} / \mathrm{mL}$ penicillin, and $100 \mu \mathrm{g} / \mathrm{mL}$ streptomycin. Competition with the functional blocking anti-TLR2 antibody (anti-CD282-PE, clone T2.5; Ebioscience) was assessed with either different concentrations of SSL3 (as a control) or different dilutions of S. aureus supernatant, which were harvested after overnight culture in THB from the WT, SSL3/4-KO, and pLukM-SSL3 strains. Anti-TLR2 antibody binding was determined by flow cytometry (FACSVerse; BD Biosciences). 
Mice and Infections

Specific pathogen-free, 8- to 12 -week-old C57BL/6 mice were purchased from Harlan-Winkelmann (Envigo, The Netherlands). The TLR2-KO mice (C57BL/6 background) were kindly provided by C. Guzmán (HZI, Braunschweig, Germany). The mice were inoculated with $4 \times 10^{7} \mathrm{CFU}$ of $S$. aureus strain SH1000 in $0.2 \mathrm{~mL}$ of $\mathrm{PBS}$ via a lateral tail vein. Infected mice were killed by $\mathrm{CO}_{2}$ asphyxiation $24 \mathrm{~h}$ after infection, and the amount of bacteria was determined by preparing organ homogenates in $5 \mathrm{~mL}$ of PBS and plating 10-fold serial dilutions on blood agar (Invitrogen, Karlsruhe, Germany). All experiments were approved by the ethics board LAVES (Niedersächsisches Landesamt für Verbraucherschutz und Lebensmittelsicherheit, Oldenburg, Germany; permit No. 33.9-42502-04-10/0296)

Mouse organ samples were spun down after homogenization, and supernatant was harvested, after which the protein concentration was determined in all samples using the Pierce BCA protein kit (Thermo Fisher Scientific) before mass spectrometry was performed.

\section{Cytokine Measurements}

IL-1 $\alpha$, IL-6, IFN $\gamma, \mathrm{KC}$, and TNF $\alpha$ levels were determined at the MultiPlex Core Facility of the Laboratory of Translational Immunology, Utrecht, using a murine 5-plex kit (BioRad). Organs were homogenized as described previously, and the supernatant was used directly for Luminex analysis.

\section{Growth Curves}

All bacterial strains were grown overnight in THB. The following day, cultures were diluted 1:100 in fresh THB and grown for $2 \mathrm{~h}$ until logarithmic phase. $\mathrm{OD}_{660}$ was measured, and bacteria were diluted to an OD of 0.01 and plated out on 96-well flat-bottom plates. $\mathrm{OD}_{660}$ was determined every $10 \mathrm{~min}$ with intermittent shaking at $600 \mathrm{rpm}$ for $10 \mathrm{~h}$ in a Fluostar Omega plate reader (Labtech).

\section{Mass Spectrometry Analysis of SSL3 Production in vivo}

Recombinant purified SSL3, protein extracts from mouse organs, and pooled normal human plasma (Innovative Research) were denatured with $10 \mathrm{M}$ urea in $100 \mathrm{mM}$ ammonium bicarbonate (Sigma-Aldrich). Subsequently, the samples were submitted to insolution digestion. First, the protein samples were reduced with $5 \mathrm{mM}$ TCEP (Tris[2-carboxyethyl]phosphine) (Sigma-Aldrich) at $37^{\circ} \mathrm{C}$ for $60 \mathrm{~min}$. Then, $10 \mathrm{~mm}$ of 2 -iodoacetamide was added to the samples. Samples were incubated in the dark for $30 \mathrm{~min}$ and diluted $5 \times$ in $0.1 \mathrm{M}$ ammonium bicarbonate before trypsin digestion $(2.5 \mathrm{ng} / \mu \mathrm{L})$ was performed overnight at $37^{\circ} \mathrm{C}$. The next morning, $10 \%$ formic acid (FA) was added until $\mathrm{pH}$ reached $2-3$ to terminate the reaction. For all dilutions, HPLC grade water was used (Sigma-Aldrich). The C18 peptide cleanup protocol was applied afterwards using Harvard Apparatus Macro SpinColumn Silica C18 (\#744101) according to the manufacturer's protocol. Samples were reconstituted in $2 \%$ acetonitrile/ $0.1 \% \mathrm{FA}$ in water.

Peptides derived from purified SSL3 were analyzed with datadependent mass spectrometry (MS) to generate a spectral library as previously described [30] using a search database containing the SSL3 amino acid sequence (UniProtKB-Q2G0X7). From the spectral library, selected reaction monitoring MS (SRM-MS) assays for all detected SSL3 peptides were generated with Skyline [31]. SSL3 peptides were spiked in human plasma digests in a concentration range from $\sim 100 \mathrm{fmol}$ to $\sim 5 \mathrm{amol}$ on column. The three SSL3 peptides (AYYTKPSFEFEK, YSVGGITK, and FMNVIPNR) with the lowest limit of detection, most linear range, and compatibility with quantitative MS workflows (Peptide Synthesis and Proteotypic Peptide Analyzing Tool; Thermo Scientific) were selected and synthesized as stable isotope-labeled peptides (AQUA peptides; Thermo Scientific). These AQUA peptides were subsequently spiked into mouse organ protein extracts before digestion at a concentration of $0.5 \mathrm{fmol} / \mu \mathrm{g}$ mouse protein. Peptide retention time normalization was performed as previously described [32].

The SRM measurements were performed on a TSQ Vantage triple quadrupole mass spectrometer (QqQ) or on a TSQ Quantiva QqQ (both from Thermo Scientific). Chromatographic separation of peptides was performed with Easy-nLC II systems (Thermo Scientific). Peptides were loaded with a constant pressure rate of 280 bar onto the analytical columns, either PicoChip (PCH7515105H354-FS25; New Objective) or EasySpray (ES802; Thermo Scientific) with $10 \mu \mathrm{L} 97 \%$ solvent A (0.1\% FA in water) and $3 \%$ solvent $\mathrm{B}(0.1 \% \mathrm{FA}$ in acetonitrile). The peptides were eluted with a linear gradient from $92 \%$ solvent A and $8 \%$ solvent B to $35 \%$ solvent $B$ over $10 \mathrm{~min}$ or $34 \mathrm{~min}$ with a constant flow of $300 \mathrm{~nL} / \mathrm{min}$. The raw data were acquired using the Xcalibur software (Thermo Scientific), processed, and analyzed with Skyline (SRM analysis software) [31] with manual validation and inspection of the results. This included retention times, similarity between library spectra and acquired data (dotp), and similarity of relative fragment ion intensities ratios between heavy and light peptide signals (rdotp).

\section{Statistical Analysis}

Statistical analysis was performed in Prism GraphPad. The data for Figures 4a and 5 were analyzed with one-way ANOVA followed by the Tukey multiple-comparison test to compare between groups. To determine statistical significance in Figure $4 \mathrm{~b}$, an unpaired $t$ test was performed.

\section{Results}

\section{Antibodies against SSL3 and SSL4 Are Detectable in} Human Serum

Humans are often exposed to $S$. aureus, either during colonization or infection [3]. Furthermore, the ssl3 and ssl4 genes are present in almost all staphylococcal strains and clinical isolates $(76-100 \%$ for SSL3 $[24,25,33,34]$ and $88-96 \%$ for SSL4 $[24,33,34])$. To determine whether SSL3 is expressed by $S$. aureus at some point during infection or colonization of the human host, we screened the serum from 36 healthy donors for the presence of antiSSL3 antibodies by ELISA (Fig. 1a). Measurable antibody levels against SSL3 were detected in all 36 donors, with an average log titer of 3.2 as determined by frequency distribution (Fig. 1b). Similar results were obtained for SSL4 antibody titers (average log titer of 3.6; Fig. 1c, d). Taken together, these data suggest that SSL3 and SSL4 are indeed 
Fig. 1. Levels of anti-SSL3 and anti-SSL4 antibodies in human sera. a, c Concentration ranges of 36 human serum samples were added to $10 \mu \mathrm{g} / \mathrm{mL}$ SSL3 (a) or SSL4 (c) coated on an ELISA plate, after which amounts of bound IgG were detected by goat anti-human IgG-PO. b, d Frequency distribution of anti-SSL3 IgG (b) or antiSSL4 IgG (d) titers defined as the log dilution reaching $50 \%$ of the OD value (from a or c) after subtraction of the background. The mean log titers are 3.2 (SSL3) and 3.6 (SSL4), meaning the serum could be diluted $\times 1,585$ and $\times 3,981$, respectively, to reach $50 \%$ of the OD value.

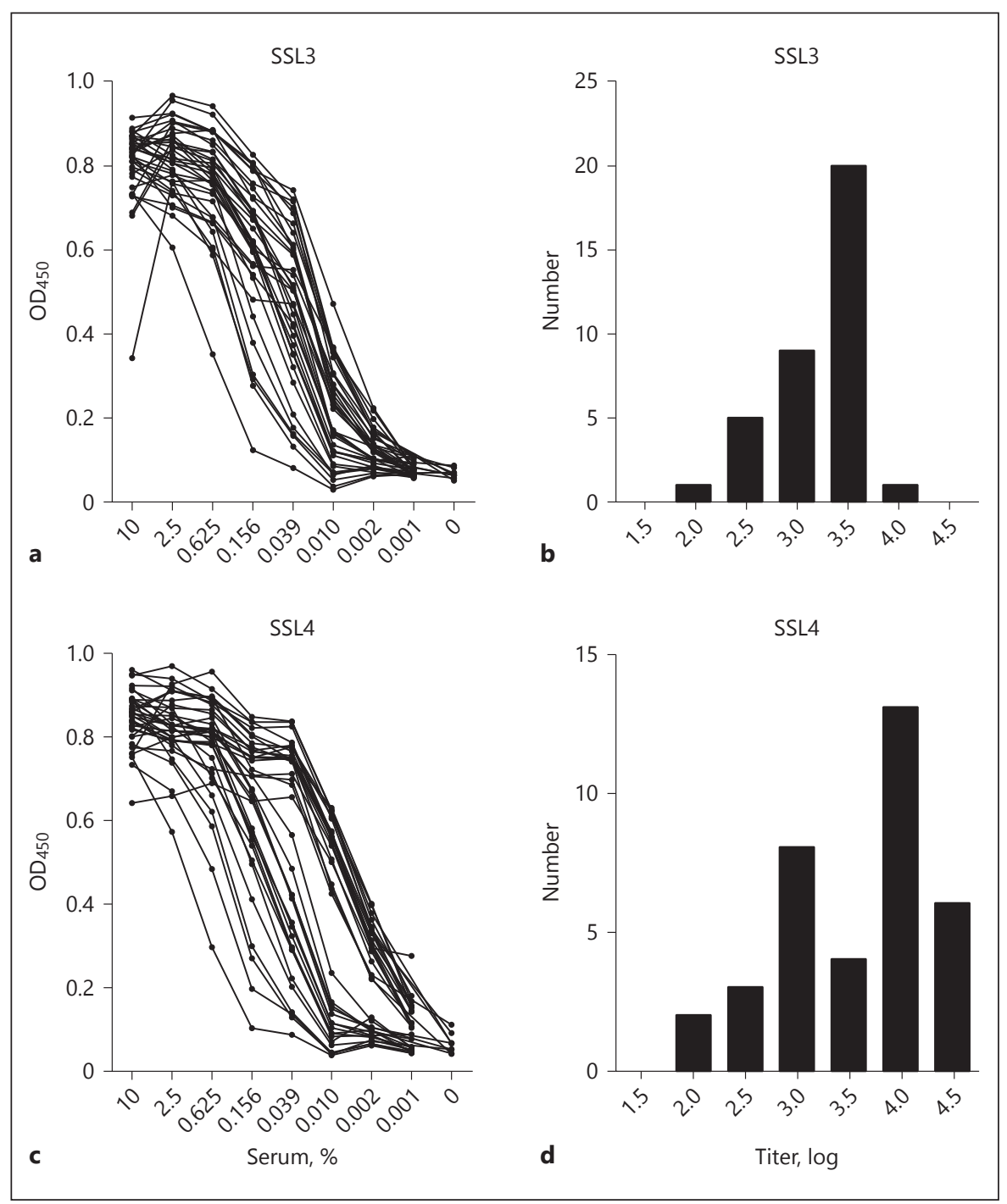

expressed, produced, and secreted by the majority of $S$. aureus strains in the human host at considerable levels and indicate a role for SSL3 and SSL4 in staphylococcal pathogenesis in vivo.

\section{S. aureus SSL3 Expression Is Low during Both in vitro} Growth and in vivo Infection of Mice

Ssl expression is highly complex and under the control of several regulatory systems. Little is known about the regulation or expression of $s s l 3$ or ssl 4 specifically. Since SSL3 is the more potent TLR2 inhibitor of both SSLs, we have mainly focused on investigating SSL3 within this study. Through the use of different techniques, including promoter reporter systems and protein detection in culture supernatant, we found that SSL3 is not expressed by
S. aureus SH1000 during in vitro growth conditions (data not shown). This suggests that induction of SSL3 production by $S$. aureus might require very specific conditions, which are difficult to mimic in in vitro culture systems. As murine TLR2 is recognized by SSL3 [17], the murine system provides a suitable model to study SSL3 during in vivo infection. However, in pilot experiments using C57BL/6 mice, we found that $S$. aureus deficient in the expression of SSL3/4 (SSL3/4-KO, created by allelic replacement) exhibited comparable levels of virulence as the parental WT strain did (Fig. 2a). This could either indicate that SSL3 and SSL4 are expressed but not required for staphylococcal virulence in the mouse model or that they are not expressed at all in this system. Transcriptional data extracted from a large RNA-Seq screen per- 


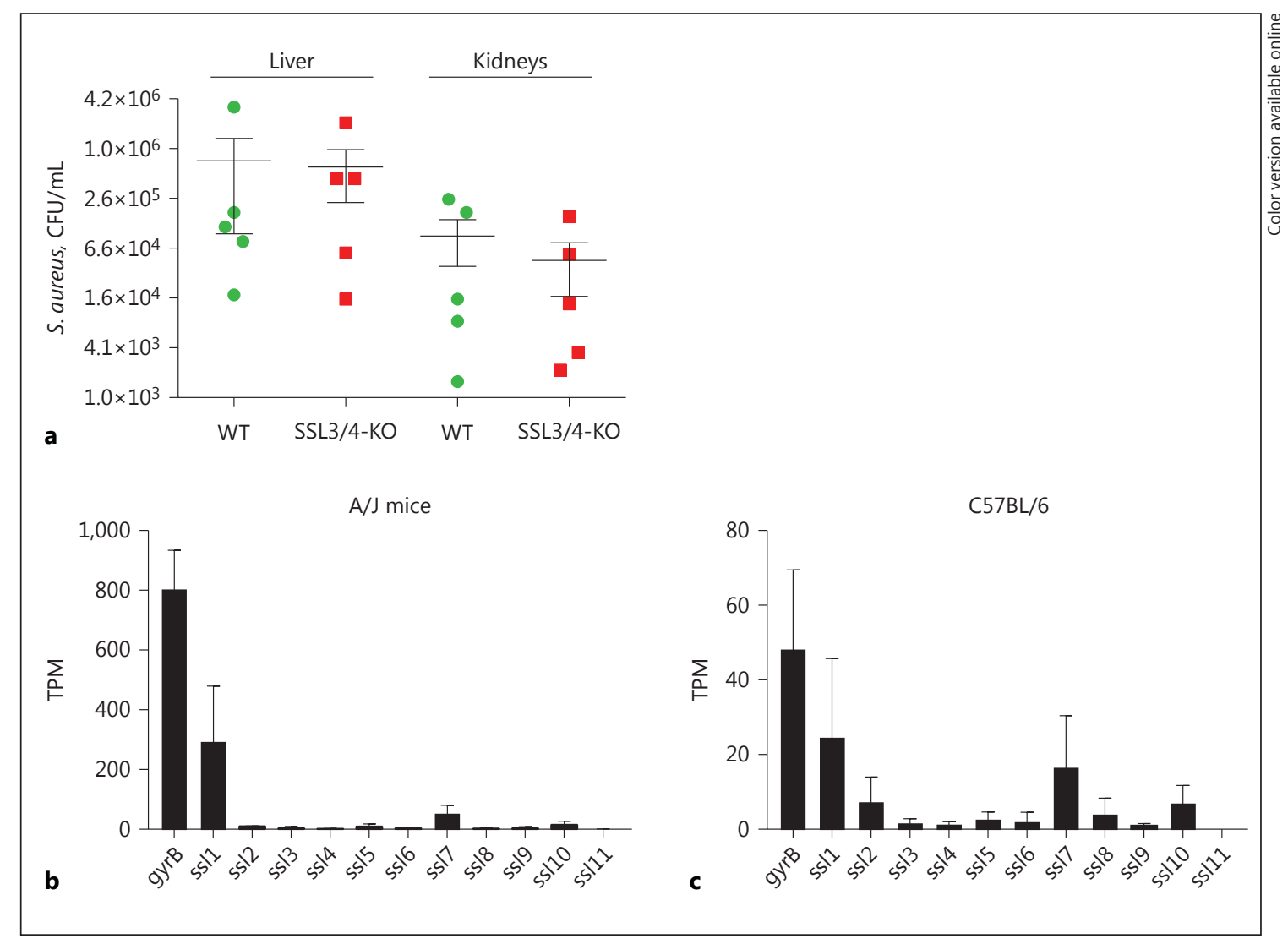

Fig. 2. SSL3 and SSL4 are likely not expressed in the murine system. a Pilot infection experiment shows no difference between wild-type (WT) and SSL3/4-knockout (KO) SH1000 strains. C57BL/ 6 female mice were intravenously injected with $4 \times 10^{7} \mathrm{CFU}$ of $S$. aureus SH1000 WT (green circles) or SSL3/4-KO (red squares). CFU (shown on a $\log _{2}$ scale) were determined in the liver (left) and kidney (right) $24 \mathrm{~h}$ after infection. Each dot represents the mean $\pm \mathrm{SE}$ of 1 mouse. b, c Expression levels of genes encoding SSL proteins

formed by Thänert et al. [35] in two different mouse strains, using an infection model and bacterial strain similar to our study (http://www.ebi.ac.uk/ena/data/ search?query=PRJEB14649), revealed that in the kidney of mice infected with S. aureus SH1000 no ssl3 or ssl4 expression could be detected (Fig. 2b, c), making the latter hypothesis likely. Thus, the environmental conditions required for efficient expression and production of SSL3 (and SSL4) by $S$. aureus might not be met in the murine infection model. This could explain our previous results and complicates further in vivo studies in mice with WT and SSL3/4-KO S. aureus strains. by S. aureus SH1000 in the kidneys of A/J (b) and C57BL/6 (c) mice $48 \mathrm{~h}$ after infection. The level of expression of the gene encoding the housekeeping protein DNA gyrase B subunit $(g y r B)$ is included for comparison. Each bar represents the mean transcripts per million $(\mathrm{TPM}) \pm \mathrm{SD}$ of triplicates. Transcription data have been extracted from the RNA-Seq data set deposited at http://www.ebi.ac.uk/ena/ data/search?query=PRJEB14649, and the corresponding experimental information has been published by Thänert et al. [35].

Generation of an S. aureus Strain Overproducing SSL3

To induce SSL3 expression by S. aureus in the murine system and circumvent the discussed limitations, an $S$. aureus strain able to constitutively produce high levels of SSL3 was generated. To develop this strain, the highly active promoter of a secreted staphylococcal toxin, the leukocidin LukMF', was used. This promoter has been reported to be constitutively active under different culture conditions [28]. The LukM promoter was cloned upstream of the $s s l 3$ gene, and this plasmid was electroporated into S. aureus SH1000 (Fig. 3a). It was confirmed that WT, SSL3/4-KO, and pLukM-SSL3 bacterial strains grow equally well when cultured overnight (online suppl. Fig. S1; see www.karger.com/doi/10.1159/000479100 for all 


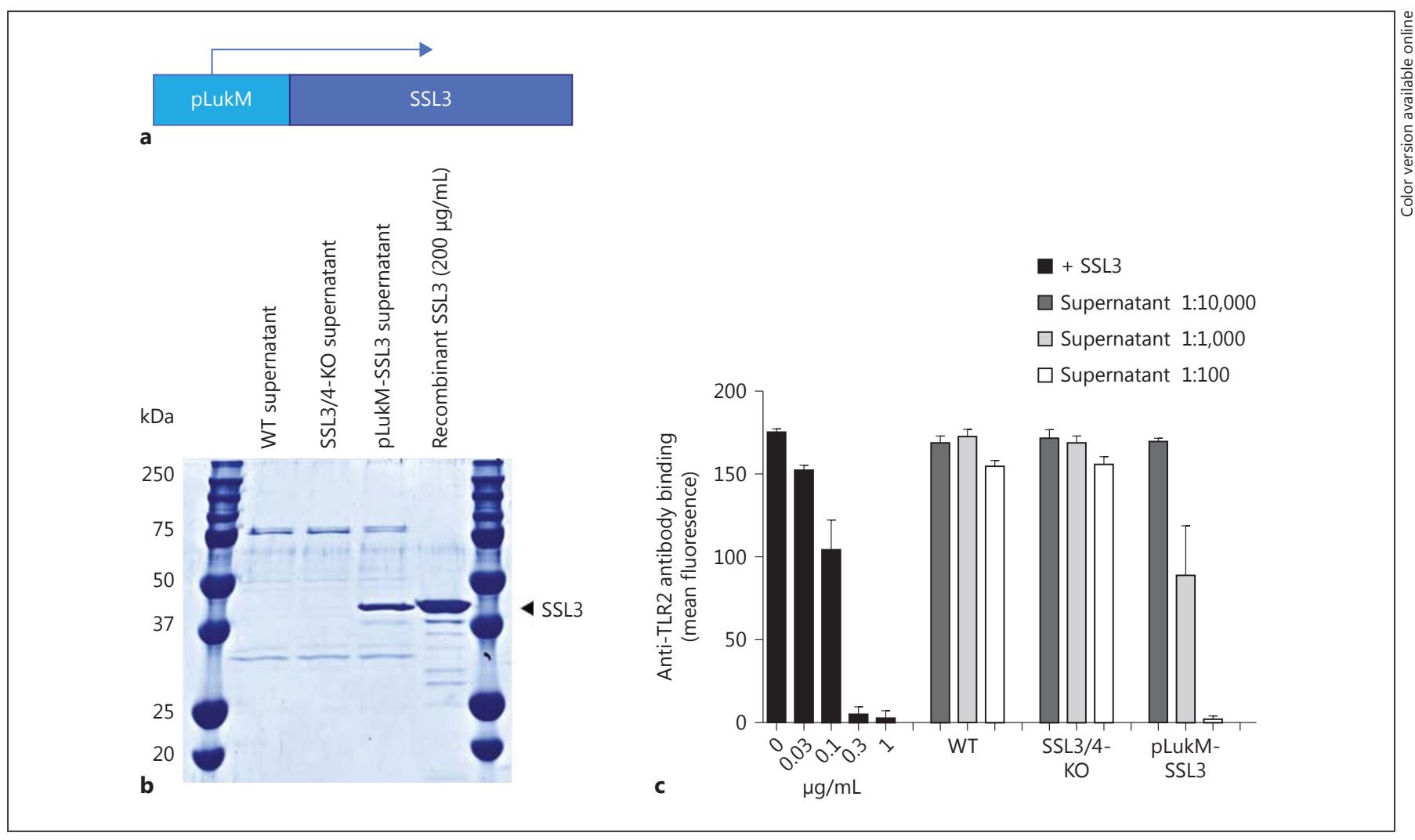

Fig. 3. Generation of the SSL3-overproducing strain. a The pLukMSSL3 DNA construct. The LukM promoter was cloned upstream of the $s s l 3$ gene. This plasmid was subsequently electroporated into S. aureus SH1000. b Supernatant of wild-type (WT), SSL3/4knockout (KO), and pLukM-SSL3 SH1000 strains (after overnight growth) was loaded on 10\% SDS-PAGE gel. Bands were visualized with InstantBlue. c An anti-TLR2 antibody competition assay [17] was performed to determine functional SSL3 levels present in the supernatant of the different $S$. aureus strains. Various concentrations of purified SSL3 and different dilutions of supernatant were incubated with HEK cells expressing TLR2 for $30 \mathrm{~min}$ at $4^{\circ} \mathrm{C}$ before addition of a functional blocking anti-TLR2-PE antibody. Subsequently, the binding of the anti-TLR2 monoclonal antibody was assessed by flow cytometry. A decrease in anti-TLR2-PE binding indicates the presence of TLR2-blocking SSL3 in the supernatant. The mean \pm SD of 3 independent experiments is shown. online suppl. material). To check SSL3 expression, after overnight culture, the supernatant of all three bacterial strains was loaded on an SDS-PAGE gel. A clear band matching the recombinant SSL3 produced in E. coli was visible in the supernatant of the pLukM-SSL3 strain, but no band corresponding to SSL3 appeared in the supernatant of the WT or SSL3/4-KO strains (Fig. 3b). To determine whether the SSL3 produced by pLukM-SSL3 was functionally active, an antibody competition screen was performed. A fluorescent anti-TLR2 antibody was added to HEK293T cells stably expressing TLR2 in the absence or presence of different dilutions of bacterial supernatant. Only the supernatant of the pLukM-SSL3 strain was capable of interfering with anti-TLR2 antibody binding (Fig. 3c). From this experiment, it can be estimated that an overnight liquid culture of pLukM-SSL3 results in the production of approximately $100 \mu \mathrm{g} / \mathrm{mL}$ of functionally active SSL3.

The SSL3-Overproducing S. aureus Strain Is More Virulent than the Parental Strain in an Intravenous Challenge Model

To investigate the effect of SSL3 in vivo, C57BL/6 mice were intravenously challenged with $S$. aureus WT, SSL3/4KO, or pLukM-SSL3 SH1000 strains, and the bacterial loads were determined in liver, kidney, and heart homogenates $24 \mathrm{~h}$ after infection. The results indicate that the pLukM-SSL3 strain was significantly more virulent than WT or SSL3/4-KO strains, which was reflected by the higher bacterial loads in all measured organs, with the 
Fig. 4. Bacterial loads in systemic organs of mice infected either with Staphylococcus aureus wild-type (WT), SSL3/4-knockout (KO), or pLukM-SSL3 strains. a C57BL/6 female mice were intravenously injected with $4 \times 10^{7} \mathrm{CFU}$ of $S$. aureus WT (green circles), SSL3/4-KO (red squares), or pLukM-SSL3 (blue diamonds). CFU (shown on a $\log _{2}$ scale) were determined in the liver (upper), kidney (middle), and heart (bottom) $24 \mathrm{~h}$ after infection. A total of 12-13 animals were included per group in 3 independent experiments. Each data point represents the mean \pm SE of 1 animal. Statistical significance was determined using one-way ANOVA followed by the Tukey multiple-comparison test. ${ }^{* *} p \leq$ $0.01, * * * p \leq 0.001$, **** $p \leq 0.0001$. b C57BL/ 6 male TLR2-KO mice were intravenously injected with $4 \times 10^{7}$ WT (circles) or pLukM-SSL3 (squares) SH1000 bacteria. Organs were harvested, and CFU were determined (as in a). Data points represent the mean \pm SE of 1 animal. A total of 6 animals were included from 2 independent experiments. Significance was determined by unpaired $t$ test. ns, nonsignificant.

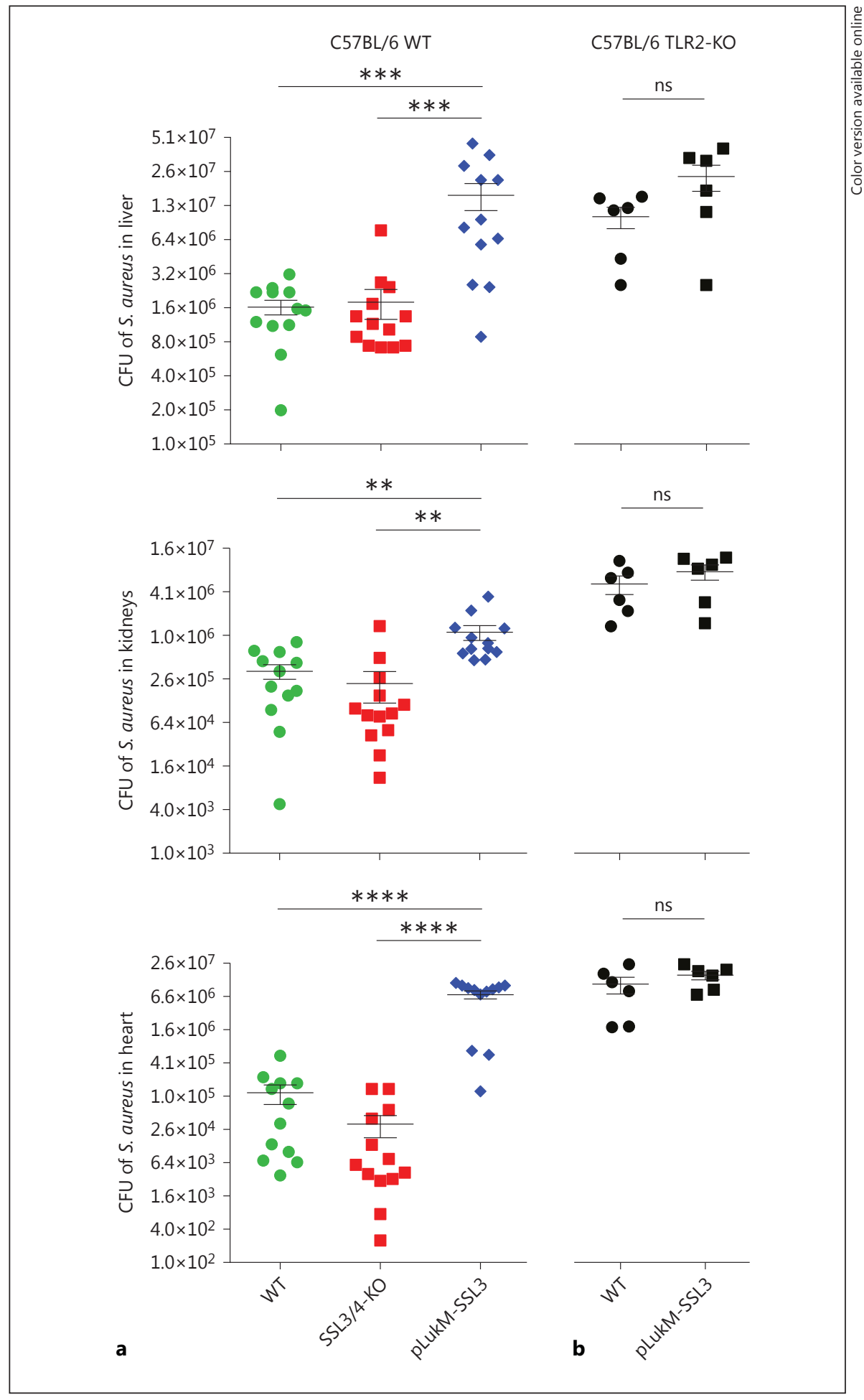

most significant differences found in the heart (Fig. 4a). As in the original pilot experiments, no significant difference was found between WT and SSL3/4-KO. Taken together, these data indicate that SSL3 expression conferred an advantage to $S$. aureus in vivo and that it is an important virulence factor when expressed. When the experiments were repeated with C57BL/6 TLR2-KO mice, no significant difference between the WT and pLukM-SSL3 


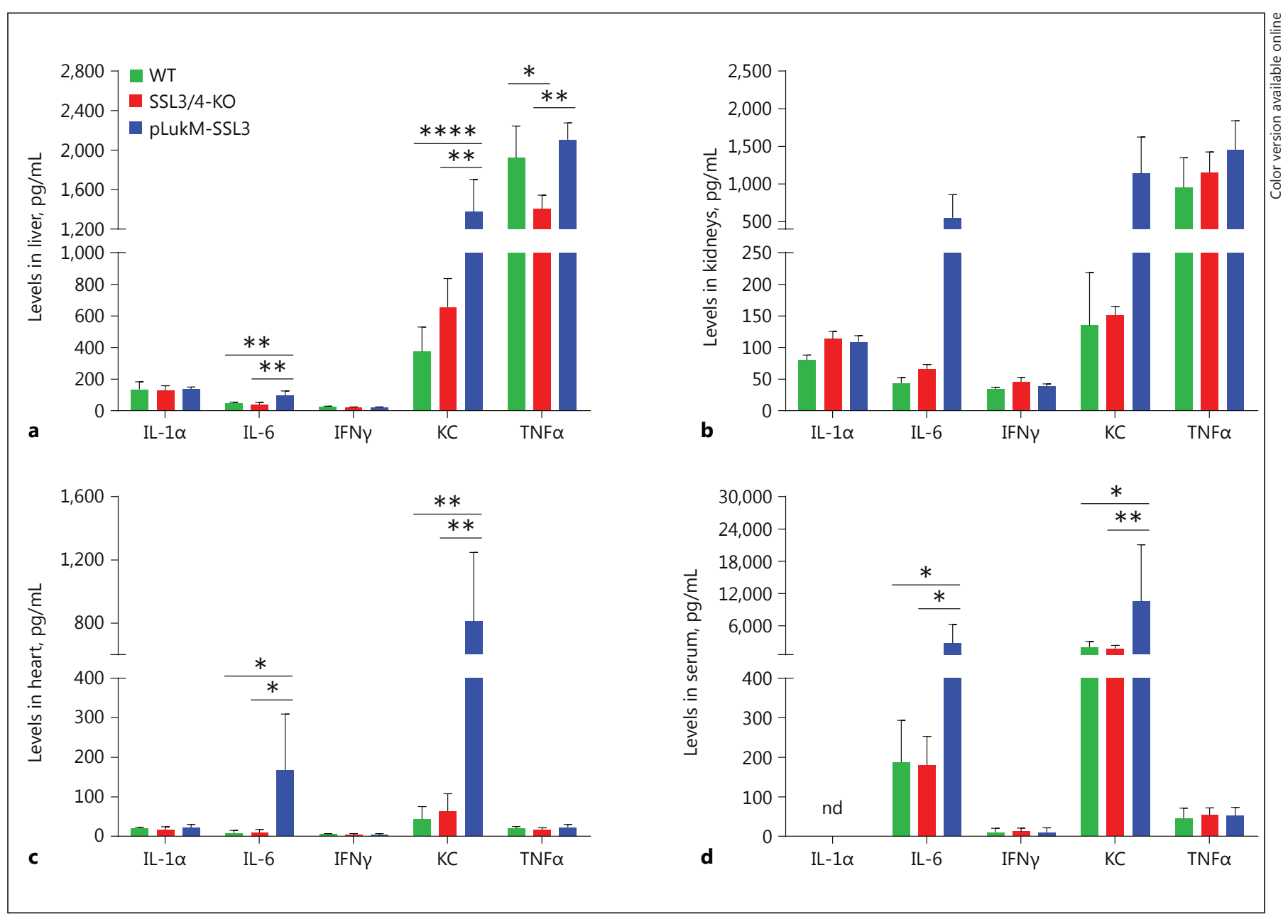

Fig. 5. Luminex analysis of produced proinflammatory cytokines in murine organ homogenates and blood. The produced IL-1 $\alpha$, IL6 , IFN $\gamma, \mathrm{KC}$, and TNFa levels were determined by Luminex analysis in the liver (a), kidney (b), and heart (c) homogenates, and sera (d) of C57BL/6 WT mice infected with Staphylococcus aureus wild type (WT) (green), SSL3/4-knockout (KO) (red), or pLukM-SSL3

(blue). For each strain, 2-4 organ samples and 8-10 serum samples were analyzed, and data points include mean and SD. Data were analyzed using one-way ANOVA followed by the Tukey multiplecomparison test (with the exception of $\mathbf{b}$ due to $n=2$ for SSL3/4$\mathrm{KO}) .{ }^{*} p \leq 0.05,{ }^{* *} p \leq 0.01,{ }^{* * * *} p \leq 0.0001$. nd, nondetectable levels.

strain was found, indicating that the enhanced virulence of the pLukM-SSL3 strain observed in the WT mice is TLR2 dependent (Fig. 4b).

Cytokine levels (IL-1 $\alpha$, IFN $\gamma$, IL-6, KC [murine IL-8 homologue], and TNFa) were determined in the homogenates of the organs (Fig. 5a-c) and in the blood (Fig. 5d) of C57BL/6 WT mice using Luminex analysis. Surprisingly, levels of IL-6 and KC were higher in the organs and serum of mice infected with the pLukM-SSL3 S. aureus strain than in those infected with the WT or SSL3/4-KO strains (Fig. 5). These results suggest that the immune stimulation due to the increase in bacterial loads found in mice infected with pLukM-SSL3 may overrule the TLR2-

dependent anti-inflammatory effects of SSL3, resulting in higher levels of inflammatory cytokines.

Detection of SSL3 Amounts Produced in vivo with MS

To examine whether actual SSL3 protein could be measured in organ samples of mice infected with WT or pLukM-SSL3 strains, we developed selected reaction monitoring mass spectrometry (SRM-MS) assays. By analyzing tryptic digests of SSL3 using shotgun spectrometry, we obtained the mass coordinates for the detectable peptide precursor and fragment ions (not shown). Next, the recombinant SSL3 peptides were serially diluted in digested human plasma and analyzed with SRM targeting 


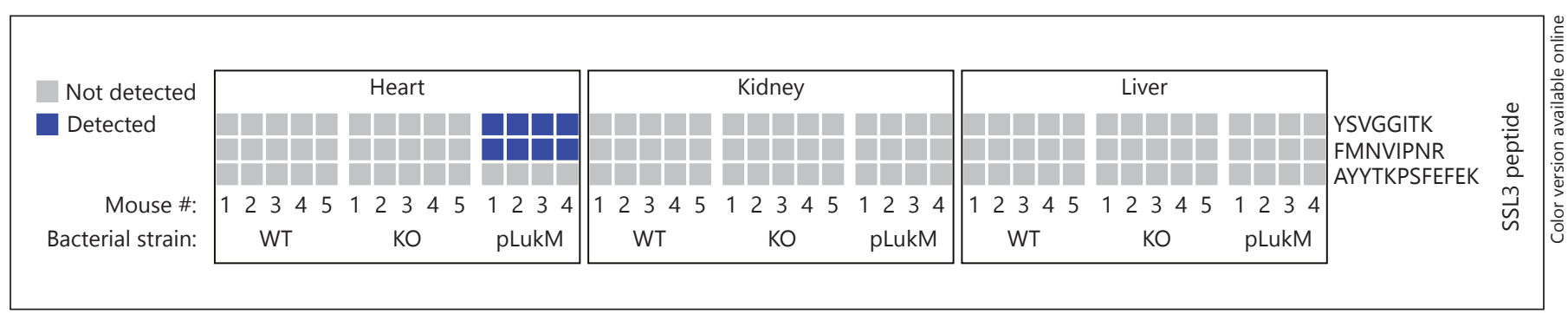

Fig. 6. Detection of SSL3 peptides in infected mouse tissues. The indicated SSL3 peptides (YSVGGITK, FMNVIPNR, and AYYTKPSFEFEK) were targeted using selected reaction monitoring mass spectrometry in tryptic digests of the heart, kidney, and liver homogenates from C57BL/6 mice infected with wild-type (WT),
SSL3/4-knockout (KO), or pLukM-SSL3 Staphylococcus aureus strains. In samples with detected SSL3 peptides (blue), the concentration was below the limit of stable isotope-labeled peptide quantification (see online suppl. Fig. S3). the SSL3 peptides previously detected (online suppl. Fig. S2). From this data set, three peptide sequences (AYYTKPSFEFEK, FMNVIPNR, and YSVGGITK) were selected based on signal intensity (see Materials and Methods for exact criteria) of which stable isotope-labeled variants were synthesized. These heavy peptides were added to the mouse organ homogenates, after which the samples were trypsin digested and analyzed for the presence of the specific SSL3 peptides, using the labeled peptides as a reference. We were able to measure SSL3 protein expression in the heart of mice that were infected with the pLukMSSL3 strain, where two out of the three peptides could be detected (Fig. 6); however, the peptide levels were well below the limit of quantification, based on a calibration curve generated by serially diluting recombinant SSL3 protein into uninfected mouse liver or kidney homogenates before trypsin digestion (online suppl. Fig. S3). In summary, the levels of detected SSL3 in mouse heart tissue of the pLukM-SSL3-infected strain was below $20 \mathrm{pg} /$ $\mu \mathrm{g}$ of total heart protein.

Interestingly, the fact that SSL3 protein could only be detected in the heart correlates with the virulence data, since the largest difference in virulence between the WT and pLukM-SSL3 strains was also measured in the heart (Fig. 4a). In liver and kidney samples, SSL3 levels were below the detection limit for all strains.

\section{Discussion}

In the current report, we investigated the relevance of $S$. aureus TLR2 inhibitor SSL3 during in vivo infection. The lack of expression of SSL3 by S. aureus in the murine system has posed an obstacle to addressing the role of
SSL3 during experimental infection in vivo. To overcome this limitation, we have generated an SSL3-overproducing strain that can constitutively produce high levels of SSL3 both in vitro and in vivo. Mice intravenously infected with this overproducing strain had significantly higher bacterial loads in systemic organs, with the most prominent differences observed in the heart. These findings indicated that SSL3 contributes to bacterial virulence in a murine infection model when expression is induced. This is, therefore, the first report showing the relevance of SSL3 during S. aureus in in vivo infection.

The fact that SSL3 acts on murine TLR2 makes in vivo studies in mice possible using the SSL3-overproducing strain generated in this study. We should keep in mind, however, that inducing overexpression of a virulence trait is artificial and does not resemble the natural situation. Human S. aureus strains are highly adapted to the human system and are not capable to easily establish infection in mice, which can partly be explained by the human specificity of many virulence factors [5], but might also be related to differential expression of virulence determinants in humans and mice. With the current lack of more suitable in vivo models, the development of this SSL3-overproducing S. aureus strain provided a valuable tool to study the effect of SSL3 in a mouse model in vivo enabling us to determine its relative contribution to bacterial virulence.

The common presence of anti-SSL3 antibodies in human sera indicates that $S$. aureus produces SSL3 during human colonization and/or infection, but when and where it is expressed remains to be determined. In this regard, it has been reported that anti-SSL3 IgG antibody levels increased in over $95 \%$ of the patients after the onset of bacteremia, indicating that $s s l 3$ is expressed by $S$. aureus during bloodstream infection [25]. Increases in anti-
570

J Innate Immun 2017;9:561-573

DOI: $10.1159 / 000479100$
Koymans et al. 
SSL3 antibody titers have also been found in osteomyelitis patients [36]. Further research should be conducted to determine whether SSL3 is important during commensalism, colonization, or pathogenesis, or if it could have a role in preventing the elicitation of an appropriate adaptive immune response against staphylococci. The combined activation of innate and adaptive immune responses is essential for effective pathogen clearage, and SSL3 could play a role herein.

It is known that ssl expression is positively regulated by the Sae two-component system [37], a regulatory system involved in the regulation and expression of many staphylococcal virulence factors [38] and of high relevance for $S$. aureus virulence $[39,40]$. The ssl promoters, including those of ssl 3 and ssl4, contain an (imperfect) consensus sequence for the response regulator SaeR (GTTAAN $_{(6)}$ GTTAA) $[19,41]$. In contrast, the two-component system Agr is described as a negative regulator of ssls $[19,37]$. These observations are not surprising considering that the Agr and Sae systems have been reported to repress each other [37]. Furthermore, the transcription factor repressor of toxins (Rot) was found to directly interact with the ssl promoter regions and to synergize with SaeR to induce ssl promoter activation $[19,21]$. This synergy is in accordance with a study showing that activation of Agr induces the expression of a regulatory RNA, RNAIII, which in turn prevents translation of Rot through its antisense function [42]. Thus, Sae-mediated inhibition of Agr results in enhanced Rot expression, whereafter Rot induces ssl expression in cooperation with Sae. However, differences in ssl expression have been described between bacterial strains, and thus the regulatory mechanisms might also vary between strains $[37,43]$. Our experiments have been performed with staphylococcal strain SH1000, an extensively studied laboratory strain, which showed no expression of SSL3 in mice in our in vivo studies. Importantly, the choice of the bacterial strain may have a strong impact on the experimental outcome, e.g., other strains could have higher basal SSL3 production in mice than SH1000. For example, the S. aureus strain Newman, which is characterized by a point mutation in the Sae twocomponent regulatory system that results in constitutive Sae activity [44], has been shown to produce enhanced levels of several virulence factors, including ssls [37]. Thus, it might be useful to examine ssl3 expression in multiple staphylococcal strains in more detail in future studies.

It has previously been described that TLR2 is important for murine survival in intravenous $S$. aureus challenge models $[11,12]$. Our data support these findings as

SSL3 Acts as a Virulence Factor in vivo overexpression of SSL3, which might directly inhibit TLR2 signaling, resulted in higher bacterial loads in the organs of infected mice when compared with WT $S$. aureus strains. Moreover, it is tempting to speculate that the increased levels of bacterial loads observed in the TLR2KO mice in respect to WT mice (Fig. 4), which are in fact similar to the CFU seen in the pLukM-SSL3 strain, might be related to the absence of TLR2 signaling. Future experiments will address this issue. Since inhibition of TLR2 by SSL3 results in blockage of TLR2 signaling, the increase in cytokine responses seen in the pLukM-SSL3infected mice was unexpected. We can speculate that, by interfering with TLR2, SSL3 may delay the early recognition of $S$. aureus by host resident innate immune defenses, thereby favoring bacterial growth. The higher bacterial loads in the pLukM-SSL3-infected mice could result in stimulation of several other pattern recognition receptors and the induction of high levels of proinflammatory cytokines.

Our method of employing a strong constitutively active promoter to induce bacterial gene expression is a valuable tool that can be used to study the effect of proteins in in vivo systems when their expression levels are low. Furthermore, by the addition of a HIS-tag to the protein, protein purification could be performed directly from $S$. aureus supernatant. This could circumvent problems, such as LPS contamination, that can arise when protein expression is performed in bacterial hosts such as E. coli. LPS contamination can be especially problematic when studying TLR signaling or when examining the effects of purified proteins in vivo, where LPS can be toxic or skew the experimental results.

A targeted MS approach was applied to investigate whether SSL3 protein production could be measured in vivo in the organs of mice infected with the three different staphylococcal strains (WT, SSL3/4-KO, and pLukMSSL3). SSL3 protein was only detectable in the hearts of mice infected with pLukM-SSL3, but the peptide levels measured were too low to allow a quantification of actual protein amounts produced. No SSL3 protein could be measured in the mice infected with WT staphylococci, which could prove the lack of expression in the murine system. However, since the SSL3 levels measured were low even in the overproducing strain and detectable in only one of three organs, it is likely that the current method does not have enough sensitivity and will have to be further optimized before firm conclusions can be drawn. Nonetheless, it was interesting that SSL3 protein could be detected in the heart of pLukM-SSL3-infected mice, where also the largest differences in bacterial loads were

J Innate Immun 2017;9:561-573 DOI: $10.1159 / 000479100$
571 
observed, indicating that SSL3 production correlates with virulence. It might seem contradictory that no SSL3 was detected in liver of pLukM-SSL3-infected mice as the bacterial loads in the liver were higher than those found in the heart (Fig. 4a, 6). It is known that the biological background complexity can influence the detectability of low abundant peptides with SRM [45]. The liver proteome is more complex than the heart proteome, as determined in a protein atlas of mouse tissues [30], which provides a technical explanation for the different detection limits depending on the mouse organ.

In summary, the current report demonstrates the practicality of using a constitutively active promoter to study a bacterial virulence factor only marginally expressed in the currently available in vivo models. Using this system, we demonstrated that SSL3 can act as a TLR2 antagonist in vivo and that SSL3 contributes to S. aureus virulence in vivo. Overall, our findings contribute to the current knowledge on host-pathogen interactions, which are relevant for our understanding of staphylococcal pathogenesis and may aid in the development of novel therapeutics.

\section{Acknowledgments}

The authors would like to thank Sabine Lehne for excellent technical assistance. This study was financially supported by the Dutch organizations for health research and healthcare innovation (The Netherlands Organization for Scientific Research, NWO), ZonMw (\#205200004to J.A.G.S.). J.M. and C.A.Q.K. were funded by the Wallenberg Academy Fellow KAW under grant agreement No. 2012.0178 and the European research council starting grant (ERC-2012-StG-309831).

\section{Disclosure Statement}

The authors declare no conflicts of interest.

\section{Author Contributions}

K.J.K., O.G., C.A.Q.K., J.M., K.P.M.K., C.J.C.H., J.A.G.S., and E.M. conceived the experiments. K.J.K., O.G., C.A.Q.K., W.S., R.T., A.B., M.V., and C.J.C.H. performed the experiments. K.J.K., O.G., C.A.Q.K., K.P.M.K., C.J.C.H., J.A.G.S., and E.M. analyzed the data. K.J.K., C.A.Q.K., and E.M. wrote the paper.

\section{References}

1 Oliveira-Nascimento L, Massari P, Wetzler LM: The role of TLR2 in infection and immunity. Front Immunol 2012;3:1-17.

2 O'Neill LAJ, Bowie AG: The family of five: TIR-domain-containing adaptors in Toll-like receptor signalling. Nat Rev Immunol 2007;7: 353-364.

3 Lowy FD: Staphylococcus aureus infections. N Engl J Med 1998;339:520-532.

4 Monaco M, Pimentel de Araujo F, Cruciani M, Coccia EM, Pantosti A: Worldwide epidemiology and antibiotic resistance of Staphylococcus aureus. Curr Top Microbiol Immunol, Epub ahead of print.

5 Koymans KJ, Vrieling M, Gorham RD, van Strijp JAG: Staphylococcal immune evasion proteins: structure, function, and host adaptation. Curr Top Microbiol Immunol, Epub ahead of print.

6 Babu MM, Priya ML, Selvan AT, Madera M, Gough J, Aravind L, Sankaran K: A database of bacterial lipoproteins (DOLOP) with functional assignments to predicted lipoproteins. J Bacteriol 2006; 188:2761-2773.

7 Schmaler M, Jann NJ, Götz F, Landmann R: Staphylococcal lipoproteins and their role in bacterial survival in mice. Int J Med Microbiol 2010;300:155-160.
8 Bubeck Wardenburg J, Williams WA, Missiakas D: Host defenses against Staphylococcus aureus infection require recognition of bacterial lipoproteins. Proc Natl Acad Sci USA 2006;103:13831-13836.

9 Mullaly SC, Kubes P: The role of TLR2 in vivo following challenge with Staphylococcus aureus and prototypic ligands. J Immunol 2006; 177:8154-8163

10 Sun Y, Hise AG, Kalsow CM, Pearlman E: Staphylococcus aureus-induced corneal inflammation is dependent on toll-like receptor 2 and myeloid differentiation factor 88 . Infect Immun 2006;74:5325-5332.

11 Takeuchi O, Hoshino K, Akira S: Cutting edge: TLR2-deficient and MyD88-deficient mice are highly susceptible to Staphylococcus aureus infection. J Immunol 2000;165:5392-5396.

12 Yimin, Kohanawa M, Zhao S, Ozaki M, Haga S, Nan G, Kuge Y, Tamaki N: Contribution of toll-like receptor 2 to the innate response against Staphylococcus aureus infection in mice. PLoS One 2013;8:e74287.

13 Miller LS, O'Connell RM, Gutierrez MA, Pietras EM, Shahangian A, Gross CE, Thirumala A, Cheun AL, Cheng G, Modlin RL: MyD88 mediates neutrophil recruitment initiated by IL-1R but not TLR 2 activation in immunity against Staphylococcus aureus. Immunity 2006;24:79-91.
14 Stenzel W, Soltek S, Sanchez-Ruiz M, Akira S, Miletic H, Schlüter D, Deckert M: Both TLR2 and TLR4 are required for the effective immune response in Staphylococcus aureus-induced experimental murine brain abscess. Am J Pathol 2008;172:132-145.

15 Kielian T, Haney A, Mayes PM, Garg S, Esen $\mathrm{N}$ : Toll-like receptor 2 modulates the proinflammatory milieu in Staphylococcus aureusinduced brain abscess. Infect Immun 2005; 73 : 7428-7435.

16 Blanchet C, Jouvion G, Fitting C, Cavaillon $\mathrm{J}-\mathrm{M}$, Adib-Conquy M: Protective or deleterious role of scavenger receptors SR-A and CD36 on host resistance to Staphylococcus aureus depends on the site of infection. PLoS One 2014;9:e87927.

17 Bardoel BW, Vos R, Bouman T, Aerts PC, Bestebroer J, Huizinga EG, Brondijk THC, van Strijp JAG, de Haas CJC: Evasion of Tolllike receptor 2 activation by staphylococcal superantigen-like protein 3. J Mol Med 2012; 90:1109-1120.

18 Koymans KJ, Feitsma LJ, Brondijk THC, Aerts PC, Lukkien E, Lössl P, van Kessel KPM, de Haas CJC, van Strijp JAG, Huizinga EG: Structural basis for inhibition of TLR 2 by staphylococcal superantigen-like protein 3 (SSL3). Proc Natl Acad Sci USA 2015;112: 11018-11023. 
19 Benson MA, Lilo S, Wasserman GA, Thoendel M, Smith A, Horswill AR, Fraser J, Novick RP, Shopsin B, Torres VJ: Staphylococcus aureus regulates the expression and production of the staphylococcal superantigen-like secreted proteins in a Rot-dependent manner. Mol Microbiol 2011;81:659-675.

20 Attia AS, Benson MA, Stauff DL, Torres VJ, Skaar EP: Membrane damage elicits an immunomodulatory program in Staphylococcus aureus. PLoS Pathog 2010;6:e1000802.

21 Benson MA, Lilo S, Nygaard T, Voyich JM, Torres VJ: Rot and SaeRS cooperate to activate expression of the staphylococcal superantigen-like exoproteins. J Bacteriol 2012; 194:4355-4365.

22 ten Broeke-Smits NJP: A closer look at the transcriptome of Staphylococcus aureus strains; PhD thesis, University of Utrecht, Utrecht, 2012, chapt 5, pp 95-125.

23 Torres VJ, Stauff DL, Pishchany G, Bezbradica JS, Gordy LE, Iturregui J, Anderson KL, Dunman PM, Joyce S, Skaar EP: A Staphylococcus aureus regulatory system that responds to host heme and modulates virulence. Cell Host Microbe 2007;1:109-119.

24 McCarthy AJ, Lindsay JA: Staphylococcus aureus innate immune evasion is lineage-specific: a bioinformatics study. Infect Genet Evol 2013;19:7-14.

25 den Reijer PM, Lemmens-den Toom N, Kant S, Snijders SV, Boelens H, Tavakol M, Verkaik NJ, van Belkum A, Verbrugh HA, van Wamel WJB: Characterization of the humoral immune response during Staphylococcus aureus bacteremia and global gene expression by Staphylococcus aureus in human blood. PLoS One 2013;8:e53391.

26 Stapels DAC, Ramyar KX, Bischoff M, von Köckritz-Blickwede M, Milder FJ, Ruyken M, Eisenbeis J, McWhorter WJ, Herrmann M, van Kessel KPM, Geisbrecht BV, Rooijakkers SH: Staphylococcus aureus secretes a unique class of neutrophil serine protease inhibitors. Proc Natl Acad Sci USA 2014;111:1318713192.

27 Bae T, Baba T, Hiramatsu K, Schneewind O: Prophages of Staphylococcus aureus Newman and their contribution to virulence. Mol Microbiol 2006;62:1035-1047.
28 Vrieling M, Koymans KJ, Heesterbeek DAC, Aerts PC, Rutten VPMG, Haas CJC, van Kessel KPM, Koets AP, Nijland R, van Strijp JAG: Bovine Staphylococcus aureus secretes the leukocidin LukMF' to kill migrating neutrophils through CCR1. MBio 2015;6:e00335.

29 Pang YY, Schwartz J, Thoendel M, Ackermann LW, Horswill AR, Nauseef WM: Agrdependent interactions of Staphylococcus aureus USA300 with human polymorphonuclear neutrophils. J Innate Immun 2010;2: 546-559.

30 Malmström E, Kilsgård O, Hauri S, Smeds E, Herwald H, Malmström L, Malmström J: Large-scale inference of protein tissue origin in gram-positive sepsis plasma using quantitative targeted proteomics. Nat Commun 2016;7:10261.

31 MacLean B, Tomazela DM, Shulman N, Chambers M, Finney GL, Frewen B, Kern R, Tabb DL, Liebler DC, MacCoss MJ: Skyline: an open source document editor for creating and analyzing targeted proteomics experiments. Bioinformatics 2010;26:966-968.

32 Escher C, Reiter L, Maclean B, Ossola R, Herzog F, Chilton J, MacCoss MJ, Rinner O: Using $\mathrm{iRT}$, a normalized retention time for more targeted measurement of peptides. Proteomics 2012;12:1111-1121.

33 Monecke S, Jatzwauk L, Weber S, Slickers P, Ehricht R: DNA microarray-based genotyping of methicillin-resistant Staphylococcus aureus strains from Eastern Saxony. Clin Microbiol Infect 2008;14:534-545.

34 Monecke S, Slickers P, Ehricht R: Assignment of Staphylococcus aureus isolates to clonal complexes based on microarray analysis and pattern recognition. FEMS Immunol Med Microbiol 2008;53:237-251.

35 Thänert R, Goldmann O, Beineke A, Medina E: Host-inherent variability influences the transcriptional response of Staphylococcus aureus during in vivo infection. Nat Commun 2017;8:14268.

36 den Reijer PM, Sandker M, Snijders SV, Tavakol $M$, Hendrickx AP, van Wamel WJ: Combining in vitro protein detection and in vivo antibody detection identifies potential vaccine targets against Staphylococcus aureus during osteomyelitis. Med Microbiol Immunol 2017;206:11-22.

37 Pantrangi M, Singh VK, Wolz C, Shukla SK: Staphylococcal superantigen-like genes, ssl5 and ssl 8 , are positively regulated by Sae and negatively by Agr in the Newman strain. FEMS Microbiol Lett 2010;308:175-184.
38 Rogasch K, Rühmling V, Pané-Farré J, Höper D, Weinberg C, Fuchs S, Schmudde M, Bröker BM, Wolz C, Hecker M, Engelmann S: Influence of the two-component system SaeRS on global gene expression in two different Staphylococcus aureus strains. J Bacteriol 2006; 188:7742-7758.

39 Liang X, Yu C, Sun J, Liu H, Landwehr C, Holmes D, Ji Y: Inactivation of a two-component signal transduction system, SaeRS, eliminates adherence and attenuates virulence of Staphylococcus aureus. Infect Immun 2006; 74:4655-4665.

40 Voyich JM, Vuong C, DeWald M, Nygaard TK, Kocianova S, Griffith S, Jones J, Iverson C, Sturdevant DE, Braughton KR, Whitney AR, Otto M, DeLeo FR: The SaeR/S gene regulatory system is essential for innate immune evasion by Staphylococcus aureus. J Infect Dis 2009;199:1698-1706.

41 Sun F, Li C, Jeong D, Sohn C, He C, Bae T: In the Staphylococcus aureus two-component system sae, the response regulator SaeR Binds to a direct repeat sequence and DNA binding requires phosphorylation by the sensor kinase SaeS. J Bacteriol 2010;192:2111-2127.

42 Boisset S, Geissmann T, Huntzinger E, Fechter P, Bendridi N, Possedko M, Chevalier C, Helfer AC, Benito Y, Jacquier A, Gaspin C, Vandenesch F, Romby P: Staphylococcus aureus RNAIII coordinately represses the synthesis of virulence factors and the transcription regulator Rot by an antisense mechanism. Genes Dev 2007;21:1353-1366.

43 Pantrangi M, Singh VK, Shukla SK: Regulation of staphylococcal superantigen-like gene, ssl8, expression in Staphylococcus aureus strain, RN6390. Clin Med Res 2015;13:7-11.

44 Schäfer D, Lâm TT, Geiger T, Mainiero M, Engelmann S, Hussain M, Bosserhoff A, Frosch M, Bischoff M, Wolz C, Reidl J, Sinha $\mathrm{B}$ : A point mutation in the sensor histidine kinase SaeS of Staphylococcus aureus strain Newman alters the response to biocide exposure. J Bacteriol 2009;191:7306-7314.

45 Gillette MA, Carr SA: Quantitative analysis of peptides and proteins in biomedicine by targeted mass spectrometry. Nat Methods 2013; 10:28-34. 